\title{
IL PROBLEMA DELLA COMUNICAZIONE LINGUISTICA A SCUOLA: IL LINGUAGGIO SCIENTIFICO E CHIMICO IN PARTICOLARE
}

\author{
BORSESE, A. \\ Istituto di Chimica Generale. Unjversità di Genova. Viale Benedetto XV, 3. 16132 Genova. Italia.
}

\begin{abstract}
SUMMARY
In this work first of all we notice the importance of linguistic communication at school and we underline the fact that every teacher, regardless of his discipline, has the problem of making himself understood.

Then, for what concernes scientific language, we give some methodological directions. Finally we give prominence to chemical language and to the role that this language could have in teaching.
\end{abstract}

\section{INTRODUZIONE}

Inchieste relative alla comprensione di termini astratti condotte nella scuola hanno rilevato che le difficoltà di comprensione erano tanto più pronunciate quanto più complesse sintatticamente erano le frasi in cui tali termini erano inseriti. Interessanti sono, a questo proposito, gli studi di Merzyn (1987) e di Cigada (1988) sul linguaggio scientifico nelia scuola, di De Mauro (1983, 1984) sul linguaggio come mezzo di emancipazione e sui linguaggi scientifici, e di Johnson (1979) sul grado di leggibilità dei testi scientifici.

L'articolo di Johnson, in particolare, descrive i risultati di ricerche miranti a stabilire la leggibilità dei testi scritti e l'età mentale in grado di comprenderli. Le misure effettuate consentono di concludere che, per essere capite, te frasi e le parole brevi richiedono un'età mentale inferiore delle frasi e delle parole lunghe e complicate.

Spesso gli insegnanti non si pongono il problema delle parole che usano, non sono sistematicamente attenti al loro modo di comunicare con gli allievi.

Questo vale prevalentemente per gli insegnanti di materie scientifiche, anche perché il linguaggio, come il senso critico, sono sempre stati considerati di competenza degli insegnanti di materie umanistiche.
Cosicché l'insegnante di scienze spesso si rinchiude nello svolgimento nozionistico dei programmi scolasti$\mathrm{ci}$, annullando l'aspetto formativo dell'insegnamento scientifico ed accettando di fatto la presunta non competenza a svolgere un ruolo di formazione culturale complessiva.

Questa situazione va superata ed i docenti di materie scientifiche debbono riappropriarsi delle riflessioni sul valore culturale della conoscenza scientifica e delle sue applicazioni. Anche se si è passati negli ultimi anni da una lingua vista in funzione della letteratura ad una lingua il cui ruolo è diventato prevalentemente quello di consentire l'apprendimento delle scienze teoriche e soprattutto applicate.

Si tratta di un fenomeno di enorme importanza di cui debbono essere consapevoli gli insegnanti di discipline scientifiche. Infatti, da un lato è ovvio che non basta conoscere una lingua per leggere un trattato scientifico in quella lingua; un conto e l'apprendimento delle strutture fondamentali, grammaticali e lessicali di una lingua e un conto è ciò che tecnicamente si chiama la "micro. lingua», cioè la lingua di specializzazione in una data scienza, la quale presuppone certamente lo studio generale di quella lingua, ma anche lo studio specifico di quel 
linguaggio scientifico, delle sue forme e dei suoi contenuti e dunque una definizione teorica di questo specifico codice, delle sue strutture, dei suoi semantismi, dei suoi meccanismi di funzionamento. Ma è pur vero, d'altro lato, che è la microlingua lo strumento che viene utilizzato nella didattica della scienza che si insegna per condurre gradualmente gli allievi alla conoscenza dei suoi capisaldi concettuali.

Noi insegnanti di discipline scientifiche dobbiamo comprendere di poter essere protagonisti di una funzione culturale importantissima, data la nostra sensibilità al codice linguistico della disciplina scientifica oggetto del nostro insegnamento: da una parte l'uso appropriato della macrolingua per far apprendere la microlingua e, dall'altra, l'uso della microlingua per ripercorrere la macrolingua cogliendo differenze, analogie, prestiti, derivazioni, ecc.

C'è, infatti, una forte interazione tra macrolingua e microlingua. $\mathrm{E}$ occorre che ciò sia messo sistematicamente in evidenza; che si rilevi che la macrolingua presta o procura alle diverse microlingue i materiali, le regole morfosintattiche, le funzioni e le procedure di discussione necessarie al toro costituirsi, che assicura alle microlingue ie "parole» che, attraverso un trattamento di depurazione-cristallizzazione semantica (daila polisemia alla monosemia, ottenuta attraverso la neutralizzazione della connotazione), diventano dei termini.

Scopo di questo contributo è rendere consapevoli gli insegnanti di discipline scientifiche che le difficoltà che gli studenti incontrano nell'acquisire concetti scientifici potrebbero essere utilmente diminuite se essi ponessero sistematicamente attenzione al ruolo che il linguaggio ha nella comunicazione. Interessanti sono, a questo proposito, gli studi di Cassels e Johnstone (1980, $1983,1984,1985$ ) che evidenziano che il problema della scarsa comprensione degli studenti delle leggi e dei principi scientifici si deve non tanto a difficoltà nell' acquisire la microlingua quanto a diff́coltà legate alla comprensione del significato delle parole del linguaggio comune in contesto scientifico.

Il significato delle parole si consolida dentro di noi via via che si creano per esse nuove connessioni con le conoscenze che già possediamo. Una parola nuova che non riesca a trovare un aggancio con conoscenze che già si possiedono resta per noi senza significato. L'elemento linguistico non puô, cioè, essere imposto ma, deve svilupparsi contestualmente all'evoluzione dei bisogni comunicativi e di pensiero degli individui.

\section{L'APPRENDIMENTO DEL LINGUAGGIO SCIENTIFICO}

Quello che si deve pretendere inizialmente dagli studenti è che essi compiano lo sforzo di usare nella maniera più appropriata, sfruttandone tutte le potenzialità, it linguaggio di cui dispongono (il linguaggio comune) e che lo facciano per iscritto.
Per risolvere il problema della registrazione dei dati dell'osservazione oggi si ricorre alla scheda strutturata delle osservazioni dove si pongono domande cui lo studente deve rispondere sulle esperienze svolte.

Attraverso la scheda si tenta di risolvere anche il problema dell'osservazione corretta, indirizzando l'attenzione degli studenti a quegli aspetti della realtà e dei fenomeni che interessano per lo studio intrapreso.

Ma nel processo didattico lo studente deve progressivamente svincolarsi da questa scheda.

Occorre che egli sia messo nelle condizioni di fare uso autonomo delle metodologie di registrazione dei dati, di imparare a fare le domande giuste al fenomeno o al problema considerato.

Tale autonomia presuppone chiara coscienza dell'oggetto di studio e delle finalità dell'attività intrapresa. E ciò perché tale consapevolezza consente di comprendere il significato del relativo linguaggio.

Infatti, essere consapevoli del fatto che lo studio delle scienze è in gran parte ricezione, uso, rielaborazione, trasmissione ed anche produzione di informazioni porta come conseguenza a convincersi della necessità deli'tuso di un linguaggio che deve avere le caratteristiche di precisione e di comprensibilità.

La verifica della validità del linguaggio usato si ha attraverso il confronto con i compagni, nel lavoro di gruppo e, soprattutto, nel confronto con l'insegnante.

II processo didattico, così come è attuato oggi, è molto spesso una comunicazione unidirezionale che va dal docente allo studente, interrotta solo dall'interrogazione o dal test scritto. Occorre, invece, che il confronto avvenga anche quando l'insegnante spiega la lezione.

Tale confronto significa che i due poli di riferimento, docente e studente, debbono trovare inizialmente un linguaggio comune su cui convergere, poiché è inđubbio che, in partenza, le forme di comunicazione sono nettamente disuguali.

Questo significa dover ricercare un indubbiamente difficile equilibrio tra forme di comunicazione-espressione propriamente individuali ed un codice comune su cui convergere.

L'insegnante deve verificare in ogni momentol'espressione usata, altrimenti rischia di far apprendere tante parole nuove ma di non far recepire assolutamente i contenuti.

Solo a partire da tale denominatore comune ha senso un intervento pedagogico teso a far evolvere il linguaggio dello studente verso una maggiore specificità, con l'introduzione di codici particolari e di regole d'uso precise.

Una importante esigenza del processo didattico è quella della consapevolezza del docente del suo diverso grado di sensibilità al codice linguistico della đisciplina che 
insegna rispetto allo studente. Questa diversa sensibilità è spesso motivo di incomprensione. Si prenda, ad esempio, la nomenclatura chimica. Per lo studente essa non è altro che la caratterizzazione formale delle sostanze, come l'uso del nome proprio per caratterizzare una persona.

Per l'insegnante essa risponde non solo ad esigenze di designazione e di rappresentazione, ma è portatrice di informazioni sulle proprietà delle sostanze, sulla loro struttura, costituisce il risultato di un numero assai vasto di osservazioni sperimentali.

Pud accadere allora che l'insegnante faccia implicitamente un uso della nomenclatura che all'allievo non è possibile comprendere, non per pigrizia mentale o perché non capisce nulla, ma perchế non possiede gli elementi per poter appieno apprezzare il valore della nomenclatura.

Altro problema di grande interesse didattico è quello relativo alla funzione della definizione, che stenta ad uscire dal ruolo di domanda-risposta per assumere quello più corretto di concetto concretamente operativo. Si tratta di precisare quali siano le condizioni che deve soddisfare un linguaggio per risultare adeguato alle esigenze di quel dato campo di ricerca, di stabilire su quali principi generali è necessario fondare il linguaggio utilizzato. $E^{\prime}$ necessario, per prima cosa, individuare la specifica angolazione con cui quella certa scienza guarda la realtà che ci circonda, rendere esplicito l'oggetto di studio della specifica scienza che si considera.

\section{IL LINGUAGGIO CHIMICO}

Facendo riferimento, in particolare, alla chimica, sappiamo che essa studia la struttura e le proprietà della materia e sappiamo che tale studio si effettua attraverso la individuazione, la classificazione e la trasformazione delle sostanze; it fine di questa scienza è quello di prevedere, controllare, progettare il decorso dei fenomeni.

Il linguaggio chimico dovrà, perciò, provvedere a descrivere le sostanze ed i fenomeni che le coinvolgono e ad esprimere le teorie ed $i$ concetti derivati da questi fenomeni.

Poiché, peraltro, la chimica ha necessità di caratterizzare le sostanze $e$ ha grosse esigenze di classificare e sistematizzare, il linguaggio dovrà avere prevalentemente caratteristiche designative.

Vediamo come i chimici hanno costruito il loro linguaggio per tentare di rispondere a queste esigenze.

Si diceva che occorre prima di tutto designare le sostanze. Per compiere questa operazione i chimici non si sono limitati a dare un nome alle cose, ma hanno voluto anche rappresentarle con simboli che ne esprimessero fedelmente la costituzione. Hanno, cioè, costruito un linguag- gio provvisto di due componenti: la nomenclatura e la simbologia.

I simboli, che sono il vero e proprio «linguaggio scritto» dei chimici, rappresentano probabilmente l'esempio più emblematico di lingua polisintetica, e questa polisinteticità può essere colta tanto più quanta più chimica si conosce; da questo punto di vista sarebbe fondamentale far soffermare l'attenzione degli studenti su come si va arricchendo per loro il significato delle formule man mano che procede l'apprendimento della chimica. Per noi chimici le formule sono strumento intellettuale ed operativo indispensabile, ma per gli studenti, quando ancora non conoscono la struttura e lo sviluppo logico e storico della chimica, cosa possono significare?

Per quanto riguarda la nomenclatura, è fondamentale rendere consapevoli gli studenti che i termini-oggetto della chimica sono stati modificati nel tempo, nel tentativo effettuato dai chimici di superare la semplice etichettatura, cercando di individuare, per i diversi oggetti, termini che non deformassero la reattà, che ne esprimessero il più possibile la costituzione, come accade per la simbologia; renderli consapevoli del fatto che il linguaggio chimico ufficiale è russcito in parte a realizzare tra nomenclatura e simbologia quella che Dagognet (1987) chiama una corrispondenza «vocostrutturale».

Solo per poche sostanze sono stati mantenuti i nomi storici.

E' necessario però anche far rilevare l'esistenza di un problema didattico legato alla persistenza dell'uso, nella nomenciatura, cioè nel denominare gli oggetti, del linguaggio chimico tradizionale sia nella divulgazione che nei testi scientifici, e della conseguente necessità di introdurre alcuni elementi essenziali di questo linguag. gio «d'uso" accanto a quello ufficiale che, peraitro, $e$, in buona misura, autoesplicativo.

Ma a scuola occorre anche spiegare che descrivere le sostanze non vuol solo dire designarle indicandone il nome, ma anche individuarne le proprietà che consentano di associare al nome un significato.

Cioè, dopo aver associato a quella particolare sostanza il nome alluminio, a quell' altra quello di idrogeno, a quell' altra ancora fosforo, e così via, ammoniaca, glucosio, metano, ecc., i chimici hanno tentato di caratterizzarle associando ad ognuna un certo numero di proprietà; per esempio, alla sostanza alluminio si sono associate la solidità, la lucentezza, il colore grigio, la capacità di condurre la corrente ed il calore, la duttilità.

In seguito si è, però, constatato che le proprietà indivi. duate come caratteristiche dell'alluminio e che si pent* sava di utilizzare per riconoscerlo e đarne la definizione erano possedute da numerose altre sostanze, per esempio ferro, cobalto, zinco, magnesio, stagno, ecc.

Si sono allora individuate altre proprietà possedute dall'alluminio nel tentativo di distinguerlo dalle sostanze che possedevano le stesse proprietà individuate precedentemente. 
Es sono riconosciuti altri comportamenti dell' alluminio, per esempio: solubilità in acidi e basi forti, capacità di comportarsi sia da acido che da base, potere riducente..., ma si è constatato che anche queste proprietà erano possedute đa più sostanze.

Proseguendo questa operazione di ricerca di proprietà distintive ci si è accorti che l'elenco di proprietà necessarie per caratterizzare una certa sostanza diventava molto lungo $\mathrm{e}$ che si rendeva necessario trovare una via per semplificare la situazione.

Si introdussero allora nuovi termini con cui indicare "pacchetti di proprietà»; si trattava di termini in qualche misura «astratti», non essendo riferibili ad alcuna singola sostanza, ma di grande utilità perché, concentrando in un solo termine un insieme di proprietà, permettevano di classificare le diverse sostanze in maniera molto più rapida e sintetica.

Così, per esempio, il termine inventato per rappresentare il pacchetto di proprietà «lucentezza, capacità di condurre la corrente elettrica ed il calore, duttilità, colore grigio, ecc." è stato quello di metallo. Termini come questo che, come si è già sottolineato, non individuano tuna sola sostanza ma un gruppo di sostanze si chiamano designanti di gruppo.

Altri designanti di gruppo sono in chimica, per esempio: ossidante, riducente, alogeno, acido, base, catalizzatore, elemento, composto, soluzione...

Si è detto che il linguaggio chimico deve anche provvedere a designare i concetti oltre che gli oggetti della chimica. Questa necessità ha condotto all'introduzione, accanto ai termini-oggetto, dei termini-concetto. Per questi termini si pone il problema di dar loro una definizione.

$\mathrm{E}$, a questo proposito, è necessario come docenti essere consapevoli, da una parte, che anche il linguaggio scien* tifico evolve nel tempo in relazione allo sviluppo della scienza e, dall'altra, che le definizioni hanno un carattere convenzionale che le rende vere solo se si conoscono con precisione i limiti della loro validità. La consapevolezza dell'evoluzione storica del linguaggio scientifico consente di comprendere che lo sviluppo della scienza chimica può far perdere significato a certi termini, può far mutare il significato di altri.

La consapevolezza dei limiti delle definizioni si raggiunge attraverso una riflessione sui contenuti della propria disciplina che appare indispensabile per chi ha iI compito di insegnarla. A proposito dell'uso della defini. zione, si prendano, ad esempio, alcune definizionj di acido:

- sostanza che corrode i metalli

- sostanza capace di fornire protoni

- sostanza composta da non metallo più acqua

- sostanza contenente un orbitale libero a bassa energia, disponibile quindi per accogliere un dippietto elettronico

- sostanza capace di accettare ioni negativi

Quale tra queste definizioni è la migliore? Non esiste una definizione migliore delle altre, il problema è un altro: si tratta di usare coerentemente la definizione che si decide di fornire.

Ma l'insegnante tende, spesso, a far uso di tutte le definizioni in ruoli intercambiabili preferenziando quelle più teoriche, perché lo aiutano meglio a spiegare i fenomeni; tale scelta lo costringe poi ad introdurre lunghe disquisizioni sulle strutture elettroniche e sulla natura del legame.

E'anche necessario che l'insegnante rilevi sempre il campo di validità della definizione usata, per far comprendere agli studenti la sua generalità o provvisorietà o insufficienza. Occorre anche, affinché una definizione assuma per lo studente reale significato concettuale, che la sua introduzjone venga preceduta da un grosso lavoro didattico. Invece capita che nei testi le definizioni siano presenti già all'inizio, e ciò può causare anche grossolani errori nella loro formulazione. Io credo che alla schematizzazione, all'astrazione di certe proprietà attraverso la definizione per evidenziarne altre e per passare al quantitativo bisognerebbe arrivare solo dopo che gli student abbiano compreso il senso delle approssimazioni, e non come punto di partenza perché in questo modo nella mente degli studenti rischía di emergere solo il contrasto con la loro esperienza quotidiana.

Per far raggiungere agli studenti i traguardi linguistici prefissati ci sono una serie dí indicazioni concrete per il docente in un contributo di Sutton (1980) che sollecita metodi di insegnamento che facilitino una maggiore esplorazione, la riscoperta e la ricreazione dei significati.

\section{CONCLUSIONI}

Concludendo queste brevi considerazioni sul linguaggio scientifico, voglio rilevare che assai raramente, per non dire mai, si mette in evidenza che tale linguaggio è una vera e propria lingua viva; non si tilevano le sue connessioni sia con i contenuti che con i metodi scientifici; non si sottolinea abbastanza che esso non è affatto riconducibile alla sola simbologia.

Da ciò consegue che il linguaggio scientifico viene inteso in maniera del tutto strumentale ed accessoria, senza alcuna dignità culturale e conoscitiva, che gli studenti sono costretti a mandare a memoria (faccio sempre riferimento, a titolo esemplificativo, al linguaggio chimico) una Iunga sequenza di termini, simboli, formule, senza essere coscienti delle implicazioni teoriche che stanno alla loro base, e che gli stessi insegnanti non sì rendono conto dell'importanza che ha l'affrontare 
il problema del linguaggio per l'acquisizione corretta e la comprensione dei contenuti.

Una diffusione delle scienze che non investa a fondo i problemi del linguaggio e dei mezzi di comunicazione ridurrebbe il problema scientifico nella scuola alla rimo- zione di tutti gli ostacoli, veri o supposti, ad un rapporto acriticamente fiduciario con gli «esperti», i soli, per definizione, in grado di valutare e decidere per il «meglio», come i filosofi platonici, come traspare dalle tecnocratiche concezioni educative di Scwabb e Brandwein (1967).

\section{RIFERIMENTI}

CASSELS, J.R.T. e JOHNSTONE, A.H., 1980. Understanding of Non-technical words in science. (Royal Society of Chemistry: London).

CASSELS, J.R.T. e JOHNSTONE, A.H., 1983. Education, in Chem., 20, p. 10.

CASSELS, J.R.T. E JOHNSTONE, A.H., 1984. Journal Chem. Education, 61, p. 613.

CASSELS, J.R.T. e JOHNSTONE, A.H., 1985. Words that matter in science. (Royal Society of Chemistry: London).

CIGADA, S., 1988. Il Linguaggio delle scienze ed il suo insegnamento. (Ed. La Scuola: Brescia).

DAGOGNET, F., 1987. Tavole e Linguaggi della Chimica. (Theoria: Roma).
DE MAURO, T., 1983. Il linguaggio come mezzo di emancipazione, Scuola e Città, 9, p. 376. (La Nuova Italia: Firenze).

DE MAURO, T., 1984. Linguaggi scientifici, Riforma della Scuola, p. 29. (Ed.Riuniti: Roma).

JOHNSON, R.K., 1979. The School Science Review, 212, p. 562.

MERZYN, G., 1987. The language of school science, Intermational Journal of Science Education, 4, p. 483.

SUTTON, C., 1980. La scienze, it linguaggio, il significato, The School Science Review, 218, p. 47.

SCWABB, J. e BRANDWEIN, P., 1967. L'insegnamento delle scienze. (Armando: Roma). 